\title{
"ANDANDO PELOS SERTÕES": INTENÇÕES DE MOBILIDADE EM ÁREAS URBANAS DIANTE DAS SECAS NO SERIDÓ POTIGUAR
}

\author{
"Walking through the sertões": mobility intentions in urban areas \\ against the droughts in the Seridó Potiguar
}

Isac Alves Correia*

\begin{abstract}
Resumo. Através de uma pesquisa domiciliar urbana de 2017 no Seridó Potiguar (Rio Grande do Norte, Brasil), esse artigo busca entender como a intenção de mobilidade pode diferir entre os indivíduos, de acordo com suas experiências de mobilidade, o sexo e a idade. A fonte de dados compreende uma amostra probabilística em três estágios e representativa para a população urbana do Seridó Potiguar. A análise dos dados consiste em estatística descritiva e teste de diferença entre proporções. Os principais resultados mostram que os indivíduos que percorrem distâncias mais longas consideram mais a mobilidade por causa da seca que os demais grupos e maior proporção de indivíduos com intenção de se mover nos grupos etários de 20-24 e de 25-34 anos. Esse estudo contribui com a literatura ao fornecer uma análise sobre a intenção de mobilidade e os seus determinantes como o tempo de permanência fora do domicílio de residência habitual.
\end{abstract}

Palavras-chaves: migração ambiental; deslocamentos da seca; intenções de mobilidade.

\begin{abstract}
Through a urban home survey in 2017 in Seridó Potiguar (Rio Grande do Norte, Brazil), this article seeks to understand how the intention of mobility can differ among individuals, as well as in the face of attributions such as his experience of mobility, sex and age. The database is based probability sampling in three stages and representative for the Seridó Potiguar urban population. The data analyses consists of descriptive statistics and Qui-square test. The main results show that individuals who travel longer distances consider mobility due to longer distances consider mobility due to drought more than the other groups and greater proportion of individuals intending to move in the age groups of 20-24 and 25-34 years. This study contributes to the literature by providing an analysis about mobility intent and your determinants such as the length of time the individual stays away from their usual residence.
\end{abstract}

Keywords: environmental migration; drought displacement; mobility intentions.

Doutorando em Demografia pelo Centro de Desenvolvimento e Planejamento Regional (Cedeplar/ UFMG). Belo Horizonte, MG, Brasil. E-mail: isaccorreia@cedeplar.ufmg.br. Orcid: 0000-00019495-2325. 


\section{Introdução}

A literatura internacional sobre migrações e mudanças ambientais tem avançado bastante em entender como as populações respondem a esses fenômenos, especialmente falando da migração como estratégia de adaptação (Henry, Schoumaker, Beauchemin, 2004; Black et al., 2011; Gray, Mueller, 2012; Koubi et al., 2016). Por outro lado, os estudos apresentam resultados conflitantes e pouca preocupação foi direcionada a entender como o tempo e o espaço em que essas formas de mobilidade humana são praticadas interagem com a percepção ou como eles são escolhidos em função da mudança ambiental (Nawrotzki, DeWaard, 2016). Por esse motivo, esse trabalho analisa formas de mobilidade que levam em conta essas particularidades.

Em se tratando das secas, alguns estudos mostram que as migrações podem ser acentuadas como uma forma de ampliar o portifólio de capitais das famílias, com o acesso a mercados de crédito e ocupações urbanas que não estejam relacionadas ao fenômeno ambiental, com o intuito de diversificar os riscos (Sherbinin et al., 2008). Dentre outras evidências, existem resultados que apoiam essa relação para a Etiópia (Gray, Mueller, 2012), Equador (Gray, Bilsborrow, 2013), África do Sul (Hunter et al., 2014), Paquistão (Mueller, Gray, Kosec, 2014) e México (Nawrotzki, DeWaard, 2016).

Por outro lado, outros estudos consideram que os efeitos das mudanças ambientais interagem com condições socioeconômicas. Assim, as secas podem intensificar processos de exclusão e deteriorar os recursos disponíveis, inclusive os necessários para arcar com os custos da mobilidade. Por exemplo, as secas podem prejudicar as expectativas econômicas dos indivíduos, reduzir o investimento em educação e o estoque de capital financeiro das famílias, recursos que são essenciais à mobilidade. Essa deterioração dos recursos, por sua vez, torna os indivíduos menos móveis ao intensificar os riscos de migração via custos de deslocamentos. Essas conclusões se sustentam em pesquisas para o Mali (Findley, 1994), Burkina Faso (Henry, Schoumaker, Beauchemin, 2004) e México (Kniveton et al., 2008).

Vale salientar que o conceito de desastre compreende um conjunto mais amplo de riscos, tais como os choques econômicos, guerras e conflitos armados, epidemias e surtos de doenças, bem como os fenômenos relacionados ao clima, como enchentes, secas, furacões e tsunamis, ambos com impactos sobre as condições de normalidade do funcionamento social. Outra perspectiva quanto à definição de desastre abordada nesse trabalho é que eventos rotineiros podem ser excluídos, mas as mudanças na frequência e na intensidade desses fenômenos e uma grande quantidade de pessoas envolvidas são elementos suficientes para interpretá-los como desastres (Frankenberg, Laurito, Thomas, 2015).

Para o Brasil, apesar de termos pouca ou quase nenhuma evidência sobre a relação entre as estiagens e migração, os dados do The Emergency Events 
Database ${ }^{1}$ de 1940 até 2014 mostram que as estiagens foram os desastres naturais que afetaram o maior número de pessoas no País. Assim, embora a seca seja um fenômeno ambiental com um número de vítimas fatais desconhecido, foi a que sempre trouxe desafios para as instituições tanto do ponto de vista demográfico quanto econômico. Um exemplo disso é que o semiárido brasileiro, onde boa parte desses desastres está concentrada, existe uma dependência dos municípios por parte de transferências constitucionais como os Fundos de Participação dos Municípios (FPM) advindas da União, de programas como o Bolsa Família e de atividades agrícolas (Correia, 2018; Correia, Ojima, 2019).

Nos anos mais recentes (de 2011 até 2017) a população do semiárido nordestino vivenciou uma das maiores secas dos últimos 30 anos, o que comprometeu as reservas hídricas dos municípios, prejudicou a capacidade de produção agrícola e de consumo das famílias. Ademais, espera-se que as mudanças climáticas aumentem a frequência e a intensidade de eventos extremos como as secas, trazendo impactos significativos inclusive para a saúde da população, produção de alimentos e para a reprodução das suas estratégias de sobrevivência (Ojima, 2013; Ojima, Fusco, 2017; Correia, Ojima, 2019).

Um detalhe interessante é que para os indivíduos que moram no semiárido e sempre conviveram com a seca, pensar nesse fenômeno como um desastre parece contraditório. Entretanto, os períodos de secas mais severas ocorrem de forma aleatória na região, com intervalos mais ou menos de dez anos (Hirschman, 1963). Nesse aspecto, a experiência com a seca pode criar condições que se convertem em capacidade adaptativa. Para esse grupo específico a seca não seria um choque, mas uma instituição social que define papéis sociais e também o comportamento dos indivíduos e famílias em relação à mobilidade e às estratégias de sobrevivência em um sentido mais amplo. Por outro lado, reconhecer a capacidade da agência humana em responder aos desastres passa a ser relevante para entender porque algumas pessoas decidem permanecer em regiões afetadas (Gray, Mueller, 2012), mas isso não quer dizer que o desastre não afeta as condições de vida desses indivíduos.

Diante disso, o principal objetivo desse trabalho é entender como a experiência com a mobilidade, o sexo e a idade podem afetar as intenções de mobilidade nas áreas urbanas do Seridó Potiguar diante das secas. O Seridó corresponde a duas microrregiões, o Seridó Ocidental e o Seridó Oriental, ambas do estado do Rio Grande do Norte e incluídas integralmente no semiárido brasileiro (Figura 1) e no Núcleo de Desertificação do Seridó. Nelas estão inseridos 17 municípios, à saber: Acari, Caicó, Carnaúba dos Dantas, Cruzeta, Currais Novos, Equador, Ipueira, Jardim de Piranhas, Jardim do Seridó, Ouro Branco, Parelhas, Santana do Seridó, São Fernando, São João do Sabugi, São José do Seridó, Serra

EM-DAT: The Emergency Events Database - Universite Catholique de Louvain (UCL) - CRED, D. Guha-Sapir - www.emdat.be, Brussels, Belgium. 
Negra do Norte e Timbaúba dos Batistas. Essa região está integralmente inserida na Caatinga, onde a vegetação é caracterizada por árvores com aspectos secos e retorcidos, os solos são pedregosos e os rios temporários, especialmente devido às altas temperaturas, baixas precipitação e fertilidade do solo que nela predominam. Em 2010, de acordo com o Censo Demográfico, viviam nessa região em torno dos 216 mil habitantes, que dependiam basicamente de atividades agropastoris. Por outro lado, no ano de 2010, cerca de 85\% da população estava concentrada em áreas urbanas e cercadas por açudes (Morais, 2005; IBGE, 2010; Faria, 2012; Macêdo, 2012).

Figura 1 - Localização dos municípios inseridos no Seridó Potiguar (Rio Grande do Norte, Brasil), 2010

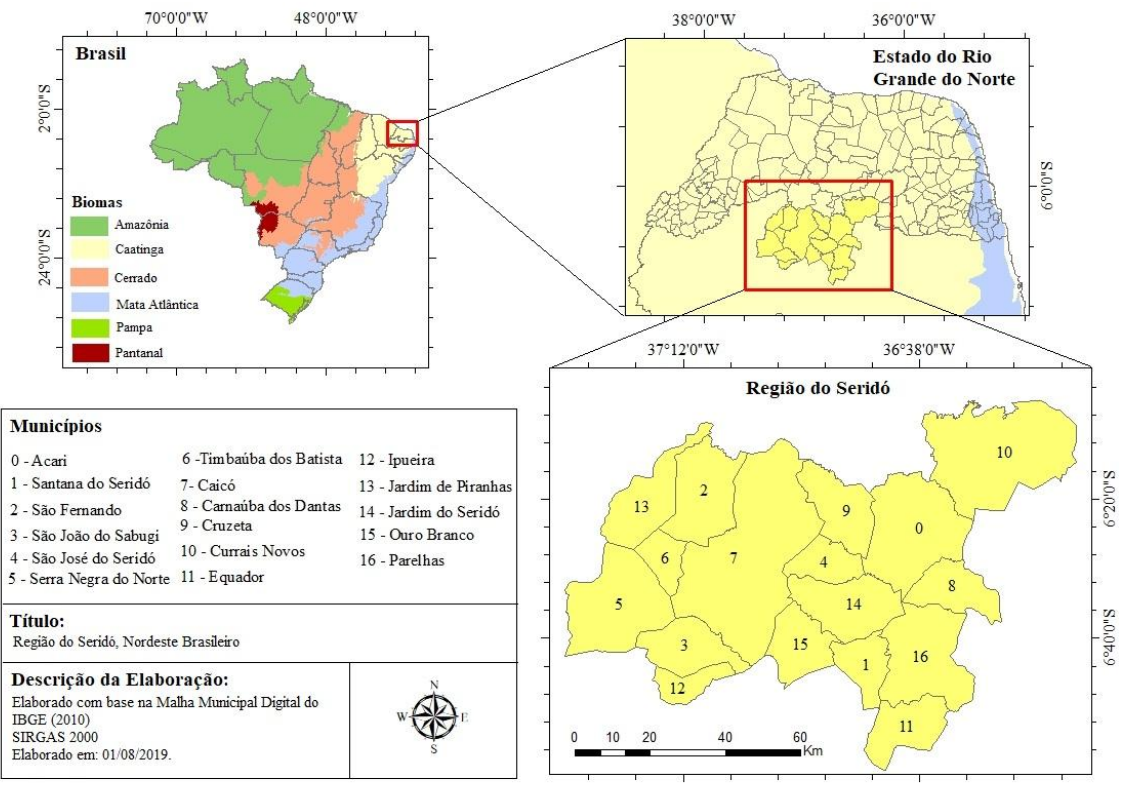

Fonte: IBGE. Malha Municipal Digital (2010).

Esse trabalho é oriundo de uma pesquisa domiciliar com representatividade amostral para a população urbana da região do Seridó Potiguar. O desenho amostral foi obtido por meio de probabilidades de seleção em três estágios (municípios, setores censitários e domicílios), conforme as metodologias descritas na seção metodológica. O survey, além de reunir um conjunto amplo de informações demográficas, socioeconômicas e ambientais e mais específicas sobre as estiagens, trata-se do primeiro do gênero e em escala micro regional para o Brasil (Barbieri et al., 2019; Correia, Barbieri, 2019). Outra relevância do trabalho é que apesar de haver uma tendência global de que os indivíduos se concentrem cada vez mais em áreas urbanas, ainda entende-se pouco sobre como as populações se adaptam 
às mudanças ambientais em contextos urbanos (Zander, Richerzhagen, Garnett, 2019), o que justifica a necessidade de estudos como esse. Além disso, maior parte dos estudos sobre migração e ambiente tem abordado as regiões da África, Ásia e do Pacífico em detrimento do semiárido brasileiro (Campos, 2015).

O trabalho está dividido em cinco seções, além dessa parte introdutória. Na seção a seguir é feita uma revisão da literatura sobre mudanças ambientais e mobilidade humana. Na terceira são explicados os procedimentos da pesquisa de campo e do desenho amostral. Na quarta são analisados e discutidos os resultados e, por fim, são feitas as considerações finais acerca dos principais resultados encontrados e das perspectivas de investigação futuras na quinta e última seção. Os principais resultados desse artigo mostraram que a intenção de mobilidade nas áreas urbanas do Seridó no contexto das secas foi mais considerada pelos indivíduos que percorreram distâncias mais longas do que os demais grupos. A pesquisa não apontou diferenças significativas na intenção de mobilidade entre homens e mulheres e houve uma maior proporção de indivíduos com intenção de se mover nas idades mais jovens, especialmente nos grupos etários de 20-24 e de 25-34 anos. O estudo concluiu que a intenção de mobilidade pode ser influenciada por fatores como o tempo que o indivíduo permanece fora do domicílio de residência habitual e que a experiência com a mobilidade poderá influenciar a intenção de mobilidade em áreas urbanas em contextos de secas no futuro.

\section{Mudanças ambientais e mobilidade}

A literatura sobre mudanças ambientais (secas, inundações, furacões, tsunames, etc) e mobilidade mostrou evidências de que os movimentos populacionais em regiões afetadas por esses desastres naturais interagem com características individuais, familiares e da comunidade, bem como com histórias locais, pressões políticas, sociais e econômicas (Black et al., 2011; Call et al., 2017). Isso emerge na dificuldade de separar questões estruturais mais abrangentes, como o desenvolvimento econômico, dos desastres ambientais (Berkes, Jolly, 2002), mas também na multicausalidade da mobilidade espacial da população (Hunter, Luna, Norton, 2015).

É possível que em contextos específicos, como situações de pobreza crônica ou vulnerabilidade social, as mudanças ambientais causem constrangimentos à migração, ao depreciar os recursos disponíveis (Black et al., 2011). Ao mesmo tempo, os estudos passaram a reconhecer a capacidade da agência humana em responder a essas mudanças, adotando estratégias de adaptação no local (Hugo, 1996; Gray, Mueller, 2012). Além disso, a migração pode ser onerosa por envolver a ruptura de laços familiares e redes de apoio e que podem ser convertidos em riscos financeiros e psicológicos (Schade, Faist, McLeman, 2015), sinalizando a 
necessidade de novas leituras e abordagens para as migrações no contexto das mudanças ambientais e de como as populações respondem aos desastres.

O modelo de Black et al. (2011) pressupõe a existência de cinco principais fatores que influenciam a migração: políticos, sociais, econômicos, demográficos e ambientais. Para os autores, a interação entre esses fatores é que cria as condições para que os indivíduos decidam migrar ou permanecer imóvel. A variabilidade em um ou mais desses cinco elementos nas escalas de espaço e tempo pode interagir de forma distinta em lugares diferentes. Isso explica o fato de que em alguns contextos as pessoas decidem deixar as regiões afetadas por mudanças ambientais enquanto outras permanecem vivendo em situações de risco ou conseguem se adaptar no local.

Só mais recentemente é que os estudiosos observaram que, no caso da seca, por exemplo, que diz respeito a um fenômeno sazonal de alta previsibilidade e do tipo slow onset, ou seja, com impactos de início lento sobre os meios de subsistência, a mobilidade laboral de curta distância aumenta (Gray, Mueller, 2012). Por outro lado, ainda não está claro por que em algumas regiões as secas aumentam a migração no sentido rural-urbano (Henry, Schoumaker, Beauchemin, 2004) e em outras não existe associação entre as mudanças ambientais e os fluxos migratórios, mas apenas uma mudança no perfil desses fluxos em relação ao sexo, idade e cor/raça (Findley, 1994). Esses achados sublinham a importância em considerar a existência de outras formas de mobilidade que não envolvem a mudança permanente de residência, como as de caráter temporário (mobilidade cotidiana/sazonal e migração de retorno) ou a diversificação entre essas formas de mobilidade e que são descritas na seção metodológica, além de entender como as secas podem intensificar processos de exclusão.

Gray e Mueller (2012) observaram que os períodos de secas severas na Etiópia aumentaram a probabilidade de migração por motivo de trabalho especialmente para os homens que viviam em famílias sem a posse da terra. De forma complementar, os autores encontraram que para as mulheres as secas moderadas diminuíram a probabilidade de casamento e de mobilidade de curta distância. Isso mostra que as mudanças ambientais podem afetar de forma diferente os grupos populacionais distintos, acentuando situações de vulnerabilidade.

A literatura sobre migrações sempre destacou o Nordeste brasileiro como uma região de expulsão populacional, especialmente até a década de 1970. Esses fluxos perderam expressividade com o aumento da participação da migração de curta distância, embora a região ainda permaneça apresentando saldos migratórios negativos altos até o último Censo Demográfico (IBGE, 2010; Correia, 2018).

O semiárido brasileiro corresponde a uma das regiões semiáridas mais povoadas do mundo, onde viviam mais de 25,9 milhões de habitantes segundo o Censo Demográfico de 2010 (Ab’Sáber, 1999; IBGE, 2010). Aliás, de acordo com o geógrafo francês Jean Dresch, "baseado nas diferentes regiões áridas 
que conhecia, podia afiançar que o Nordeste seco era a região semi-árida mais povoada do mundo" (Ab'Sáber, 1999, p. 60).

Além disso, as condições climáticas são influenciadas pelos fenômenos El Niño e La Niña, retardando os períodos chuvosos no semiárido nordestino. Estudos recentes têm argumentado que as mudanças climáticas tenderão a intensificar as estiagens em regiões semiáridas (Sellers, Gray, 2019). Como maior parte do semiárido está no Nordeste (cerca de 93\% dos 1.262 municípios, segundo a delimitação de 2017), a relação entre os fluxos migratórios e as estiagens é quase um consenso na literatura, muito embora poucas vezes as questões ambientais ocuparam lugar de destaque nos estudos sobre o tema (Ojima, 2013).

Entretanto, Gonçalves (2001) argumenta que as estiagens apenas agravam uma condição social desigual que perdura durante séculos, como por exemplo a situação fundiária, onde de um lado estão os proprietários de terra e de outro estão as famílias de pequenos camponeses. A seca de 1877 no estado do Ceará que motivou a população da cidade de Fortaleza, fazendo a população da capital mais que triplicar devido à migração, passando de cerca de 30 mil habitantes para mais de 100 mil, pode ser citada como um exemplo disso. Esse marco deu origem aos campos de concentração² da seca de 1915 e 1932 que, por sua vez, foram construídos pelo governo como estratégias políticas para que os indivíduos e famílias inteiras, castigadas pela seca severa da época e pela falta de posse da terra, não migrassem em massa para a cidade de Fortaleza e impedisse que o episódio de 1877 se repetisse. Os historiadores afirmam que as ocupações de Canudos em 1893 que deram origem à Guerra de Canudos na Bahia e do Caldeirão da Cruz do Deserto no Ceará que atraíram famílias de agricultores dos sertões da Paraíba e do Rio Grande do Norte, também foram motivadas pela situação fundiária da época (Rios, 2014).

Do mesmo modo, as emigrações nordestinas de anos mais tarde conjugaram períodos de secas (em 1951, 1958, 1970 e entre 1979 e 1983) a processos de desenvolvimento regional de forma desigual como os incentivos criados pela construção de Brasília, as ocupações da fronteira agrícola e a crescente industrialização na região Sudeste do país (Ojima, Costa, Calixta, 2014). A seca, portanto, é uma instituição social que acentua condições de vulnerabilidade social preexistentes. A sua atuação, por outro lado, é resultado de uma construção social em desarmonia nas escalas de espaço e tempo.

No semiárido brasileiro as secas estão associadas ao fenômeno El Niño e ocorre pelo menos uma seca severa a cada dez anos. A última vivenciada na região

\footnotetext{
Cabe esclarecer aqui que não havia qualquer relação entre esses assentamentos humanos e os campos de concentração nazistas. As estratégias no caso dos campos de concentração construídos no Ceará nesses períodos, muito embora tenham se associado a morte de retirantes da seca por fome e a peste, não se assemelham as práticas que culminaram no genocídio de cerca de seis milhões de judeus.
} 
durou mais de cinco anos (entre 2011 e 2017) e foi reconhecida por estudiosos como uma das mais graves dos últimos 30 anos. Em anos tidos como normais as chuvas são concentradas entre fevereiro e maio e com praticamente o resto do ano seco. Uma tendência global é que esses eventos sejam intensificados com as mudanças climáticas. O fenômeno ambiental da seca, desse modo, é presente na memória do sertanejo pela sua experiência com o passado, antecipando a sua materialização. Isso tem impacto na capacidade adaptativa à medida que as perdas são projetadas, ainda que a capacidade institucional (onde os programas de transferência de renda como o Programa Bolsa Família, Programa de Erradicação do Trabalho Infantil e Benefício da Prestação Continuada e as transferências constitucionais como os Fundos de Participação dos Estados e Municípios ocupam um papel de destaque ao dinamizarem um mercado consumidor e diversificarem a renda das famílias) e de resposta da população sejam limitadas (Gonçalves, 2001; Correia, Ojima, 2019; Barbieri et al., 2019).

As evidências entre migração humana e desastres ambientais estão aumentando (Nawrotzki, DeWaard, 2016), porém as pesquisas têm avançado pouco em entender como tempo e espaço da mobilidade podem estar associados com as percepções sobre o desastre ambiental ou o tipo de desastre. Koubi, Stoll e Spilker (2016) exploraram as percepções de migrantes e não migrantes sobre diferentes tipos de eventos ambientais, ou seja, desastres do tipo slow-onset (início lento) e sudden onset (início rápido) no Vietnã. O trabalho dos autores se constitui em uma contribuição relevante nos estudos de migração ambiental ao instigar sobre como a natureza do desastre ambiental pode condicionar as decisões de migração via percepção (Koubi, Stoll, Spilker, 2016; Koubi et al., 2016). Contudo, o avanço em compreender como essas percepções podem instigar diferentes formas de mobilidade é limitado.

Além das questões que foram aqui expostas, uma contribuição importante desse trabalho é que embora o semiárido brasileiro seja uma das regiões semiáridas mais povoadas do mundo (Ab'Sáber, 1999), maior parte dos estudos sobre as influências dos choques ambientais na migração humana está concentrada na África, Ásia e no Pacífico (Campos, 2015). Outra questão é a necessidade de investigar a relação entre a seca e a escolha por formas de mobilidade além da migração. Além disso, pouco pode-se afirmar sobre como as populações se adaptam em contextos urbanos (Zander, Richerzhagen, Garnett, 2019), apesar do rápido processo de urbanização pelo qual o mundo passa.

\section{Materiais e método}

A fonte de dados é de uma pesquisa domiciliar urbana com uma amostra de 1.064 domicílios. A pesquisa é resultado do projeto Vulnerabilidade e Adaptação no Nordeste Brasileiro: Perspectivas Locais e Regionais sobre a Urbanização no Seridó Potiguar, financiado pela Rede Brasileira de Pesquisas em Mudanças Climáticas 
(Rede Clima, FINEP/MCTI) e pelo Conselho Nacional de Desenvolvimento Científico e Tecnológicos (CNPq).

A pesquisa de campo foi realizada entre janeiro e fevereiro de 2017 com o auxílio de seis bolsistas de Desenvolvimento Tecnológico e Industrial (DTI) mais dois voluntários ligados ao projeto de pesquisa supracitado. O tempo médio de cada entrevista foi de 35 minutos e o trabalho de campo teve uma duração de três semanas. A amostra de indivíduos entrevistados é representativa para a população urbana do Seridó. A metodologia adotada na amostragem foi do tipo probabilística considerando as probabilidades de seleção em três estágios (estágio 1: município; estágio 2: setor censitário; e estágio 3: domicílio).

No primeiro estágio adotou-se o método de amostragem aleatória proporcional ao número de setores urbanos do município. No segundo estágio o método foi o aleatório simples, fixando um $\mathrm{N}=20 \%$ do número de setores urbanos de cada município, permitindo maior representatividade para os municípios com maiores números de setores urbanos. No terceiro estágio foi adotada a amostragem aleatória estratificada, em que os estratos foram definidos como: 1) domicílios com renda média inferior ou igual à $R \$ 300,00$ mensais para as pessoas de 10 anos e mais (estrato 1) e 2) domicílios com renda média superior à $R \$ 300,00$ mensais para as pessoas de 10 anos e mais (Correia, Ojima, Barbieri, 2020).

Para mensurar a percepção da mobilidade como estratégia de adaptação à seca, é perguntado se o indivíduo já pensou em se mudar por causa da seca e a experiência do indivíduo com esse fenômeno, obteve-se por meio da quantidade de períodos de seca que o indivíduo passou ao longo de sua vida até a data da pesquisa. Como assume-se que a variável de experiência com a seca afeta a mobilidade e a sua percepção como estratégia de adaptação, a intenção de mobilidade pode ser resultado da sua experiência com a seca e a mobilidade. Além disso, o sexo e a idade podem afetar a percepção da mobilidade como estratégia de adaptação.

Assume-se que o tempo e o espaço da mobilidade são diretamente correlacionadas. Assim, quanto maior a distância percorrida pelo indivíduo, maior será o tempo necessário para que o mesmo retorne ao domicílio. O tempo e o espaço percorrido pelo indivíduo também podem ser relevantes para definir o acesso a recursos, capital humano e financeiro que são extremamente importantes para definir vulnerabilidade e adaptação. Uma forma de analisar o tempo da mobilidade, embora com limitações, foi perguntar o tempo (em dias) que o indivíduo costuma ficar fora do domicílio ou que residiu em outro município e se retorna diariamente para o domicílio de residência habitual.

A seca é entendida, nesse contexto, como uma construção social em que a materialização do desastre tem uma escala de atuação complexa, porém sempre presente na memória do sertanejo. A complexidade desse fenômeno, com uma atuação em desequilíbrio nas escalas espaço-temporal tem impacto sobre 
as estratégias de adaptação dos indivíduos (Correia, Ojima, 2019) e, por isso, a percepção sobre a mobilidade como estratégia de adaptação ao desastre ambiental é relevante para entender como os indivíduos podem adotar diferentes formas de mobilidade em resposta às secas. Define-se a percepção sobre a mobilidade como estratégia de adaptação à seca, nesse caso, como a intenção de se mudar por causa da estiagem. Assim, se faz necessário entender como a intenção de se mover se constitui em formas concretas de mobilidade em resposta à seca.

Analisa-se, ainda, a intenção de se mover entre indivíduos que nasceram nos municípios os quais residem e aqueles que nasceram em municípios diferentes dos de residências atuais. Além disso, para entender como as intenções de mobilidade interagem com experiência com a mobilidade, alguns conceitos foram definidos:

mobilidade cotidiana/sazonal - indivíduo que residia no Seridó em 2017 e costuma/costumava ir a outro município com frequência regular;

migrante retornado - indivíduo que residia no Seridó em 2017 e que já fixou residência em domicílio fora do município de residência habitual.

Uma limitação em adotar esses conceitos é que alguns indivíduos podem praticar ambas ou nenhuma das formas de mobilidade. Uma alternativa é analisar grupos populacionais em quatro categorias separadas: os que não praticam nenhuma das formas de mobilidade analisadas, os que efetuam só a mobilidade cotidiana/sazonal, os migrantes retornados que não praticam mobilidade cotidiana/sazonal e os migrantes retornados que praticam mobilidade cotidiana/ sazonal (ambas).

A estratégia empírica consistiu na pesquisa de campo nos municípios do Seridó e a análise de dados foi conduzida por meio de estatísticas de teste de diferença entre proporções, o teste Qui-quadrado. Esse teste investiga se dois grupos se comportam de forma semelhante em relação às características selecionadas ao observar a significância estatística na diferença das frequências relativas. Nesse sentido, a estatística Qui-quadrado revela se os indivíduos que responderam que já pensaram em se mover por causa da seca apresentam características diferentes e estatisticamente significativas dos indivíduos que nunca tiveram a intenção de se mover por causa da seca. Para uma melhor compreensão dessa estatística de teste consultar McCullagh e Nelder (1989).

\section{Intenções de mobilidade no Seridó Potiguar}

Como esperava-se a maior parte dos indivíduos afirmaram que não pensam em se mudar por causa da seca, pois raramente as pessoas irão se mover por motivos estritamente ambientais. Um ponto interessante dessa discussão é que a seca pode exaurir os recursos disponíveis, repelindo os fluxos migratórios ao invés de acentuar. Isso ocorre porque a mobilidade só é uma estratégia de adaptação para os indivíduos que possuem os recursos necessários, tais como a renda, capital humano e a existência de laços com antigos migrantes ou indivíduos que 
tiveram outras experiências de mobilidade. Como outros estudos têm sinalizado (Hugo, 1996; Gray, Mueller, 2012; Hunter, Luna, Norton, 2015), a migração é um processo multicausal onde os desastres ambientais ocupam apenas um dentre uma gama de elementos que podem influenciar a mobilidade humana, como a renda, investimento em capital humano, dentre outros. Esses elementos, contudo, são sensíveis às mudanças ambientais, dificultando uma dissociação entre esses elementos ora facilitadores ora inibidores da mobilidade (Black et al., 2011; Koubi, Stoll, Spilker, 2016; Koubi et al., 2016).

Tabela 1 - Seridó Potiguar: indivíduos entrevistados na área urbana segundo a percepção sobre a decisão de migração induzida pela seca, o município de nascimento e a forma de mobilidade praticada pelo indivíduo, 2017

\begin{tabular}{|c|c|c|c|}
\hline \multirow{2}{*}{ Nasceu neste município? } & \multicolumn{3}{|c|}{ Já pensou em se mudar por causa da seca? } \\
\hline & $\operatorname{Sim}(\%)$ & Não (\%) & \\
\hline Sim & 22,6 & 77,4 & \multirow{3}{*}{$\begin{array}{c}\text { Qui- } \\
\text { quadrado } \\
6,46^{* *}\end{array}$} \\
\hline Não & 29,8 & 70,2 & \\
\hline Missing & 0 & 100,0 & \\
\hline Forma de mobilidade & $\operatorname{Sim}(\%)$ & Não (\%) & \\
\hline Nenhuma & 19,9 & 80,1 & \multirow{5}{*}{$\begin{array}{c}\text { Qui- } \\
\text { quadrado } \\
9,05^{* *}\end{array}$} \\
\hline Mobilidade cotidiana/sazonal & 27,8 & 72,2 & \\
\hline Migrante retornado & 29,0 & 71,0 & \\
\hline $\begin{array}{l}\text { Mobilidade cotidiana/sazonal e } \\
\text { migrante retornado }\end{array}$ & 31,2 & 68,8 & \\
\hline Missing & 27,7 & 72,3 & \\
\hline Total & 26,7 & 73,3 & \\
\hline
\end{tabular}

Fonte: Survey Seridó Potiguar (2017), N=1.064 (mun. nascimento, missing=3; form. mobilidade, missing=184). ${ }^{* * *} \mathrm{p}<0.01,{ }^{* *} \mathrm{p}<0.05,{ }^{*} \mathrm{p}<0.1$.

A Tabela 1 mostra que a intenção de mobilidade se diferencia entre os indivíduos que são naturais do município de residência atual e aqueles que não o são. Para ambos os casos, os naturais $(22,6 \%)$ e os não naturais $(29,8 \%)$, há uma menor proporção dos que já pensaram em se mudar por causa das estiagens. Entretanto, há uma diferença estatisticamente significativa à 5\% entre essas duas proporções, que é corroborada pelo teste Qui-quadrado. Desse modo, há uma tendência maior que os indivíduos não naturais do município de residência tenham a intenção de se mudar por causa da seca que os naturais.

Em se tratando da associação entre os choques ambientais e a mobilidade espacial da população, os estudos de fato têm mostrado que a maior parte dos fluxos da migração ambiental ocorre nos limites nacionais (Gray, Mueller, 2012; Hunter, Luna, Norton, 2015; Koubi, Stoll, Spilker, 2016; Koubi et al., 2016). 
Contudo, na literatura sobre mobilidade humana e mudanças ambientais de um modo geral, inclusive as estiagens, são pouco discutidos como a forma de mobilidade e o tempo que os indivíduos permanecem fora de casa podem constituir-se em estratégias de adaptação às mudanças ambientais.

Além disso, praticar formas de mobilidade de curta distância parece mais uma condição de sobrevivência do que uma estratégia escolhida pelas famílias e indivíduos em contraste a mudança permanente para regiões não castigadas pela seca. Ainda de acordo com a Tabela 1, os indivíduos que experimentam formas de mobilidade de distâncias mais longas, como o migrante retornado (29\%) e os que praticam mais de uma forma de mobilidade (ou seja, os que são migrantes retornados e efetuam a mobilidade cotidiana/sazonal, 31,2\%) tendem a considerar a mudança de residência habitual por causa da seca com maior frequência que os que experimentam formas de mobilidade de curtas distâncias (a mobilidade cotidiana/sazonal, 27,8\%). Essa percepção sobre a possibilidade de mudança de residência habitual pode estar associada às diferenças observadas entre o lugar de origem e destino, sendo a mobilidade extremamente relevante para moldar a percepção dos indivíduos.

Contudo, esses dados merecem outras leituras que inclusive levem em consideração um debate mais amplo sobre oportunidades e escolhas disponíveis aos indivíduos. A literatura tem destacado a importância da mobilidade como estratégia para diversificar a renda e de proteção a riscos, inclusive os de natureza ambiental, com o acesso a mercados urbanos e reduzir situações de vulnerabilidade, por exemplo. É claro que em outros casos, porém, os que se movem podem ser mais vulneráveis na região de destino, acentuando processos de desigualdade e de exploração de recursos com o aumento da demanda por serviços ecossistêmicos (Sherbinin et al., 2008).

Além disso, a mobilidade pode ser onerosa, tanto em termos psicológicos quanto monetários, ao romper os laços e as redes de contato do indivíduo com o domicílio e a comunidade de origem. Por outro lado, os estudos sobre migração e desastres ambientais sempre deram mais atenção aos que se movem e o impacto para as regiões receptoras e menos para aqueles que desejam se mover, mas não tiveram oportunidades de sair das regiões afetadas. A Tabela 2, nesse sentido, mostra que os indivíduos que retornam diariamente para casa $(36,1 \%)$ tendem a considerar mais a mudança por causa da seca do que os que não retornam (19,6\%). Mas, os que passam mais tempo em dias fora do domicílio de residência habitual consideram menos essa alternativa de mudança permanente de residência por causa das secas. Assim, embora perfis de mobilidade puramente forçada ou voluntária seja difícil de observar na prática, cabe aqui fazer uma reflexão nessa perspectiva sobre esses resultados (Hugo, 1996; Barbieri, 2011). Ainda assim é um pouco obscuro o caráter de voluntariedade na condição de mobilidade dos indivíduos. 
Tabela 2 - Seridó Potiguar: indivíduos entrevistados na área urbana segundo a percepção sobre a decisão de migração induzida pela seca, o tempo que costuma ficar fora do domicílio e se retorna diariamente para o domicílio, 2017

\begin{tabular}{|c|c|c|c|}
\hline \multirow{2}{*}{ Tempo (dias) } & \multicolumn{3}{|c|}{ Já pensou em se mudar por causa da seca? } \\
\hline & Sim (\%) & Não (\%) & \\
\hline$<1-6$ & 40,5 & 59,5 & \multirow{6}{*}{$\begin{array}{c}\text { Qui- } \\
\text { quadrado } \\
17,38 * * *\end{array}$} \\
\hline $7-15$ & 30,5 & 69,5 & \\
\hline $16-20$ & 29,0 & 71,0 & \\
\hline $21-30$ & 21,8 & 78,2 & \\
\hline 30 e + & 22,8 & 77,2 & \\
\hline Missing & 22,6 & 77,4 & \\
\hline Retorna diariamente? & $\operatorname{Sim}(\%)$ & Não (\%) & \\
\hline Sim & 36,1 & 63,9 & \multirow{3}{*}{$\begin{array}{c}\text { Qui- } \\
\text { quadrado } \\
10,46^{* *}\end{array}$} \\
\hline Não & 19,6 & 80,4 & \\
\hline Missing & 25,7 & 74,3 & \\
\hline Total & 26,7 & 73,3 & \\
\hline
\end{tabular}

Fonte: Survey Seridó Potiguar (2017), N=1.064 (retorna diariamente, missing=746; tempo, misssing $=380)$. ${ }^{* * *} \mathrm{p}<0.01,{ }^{* *} \mathrm{p}<0.05,{ }^{*} \mathrm{p}<0.1$.

A proporção dos que já pensaram em se mudar por causa da seca é maior para mulheres $(27 \%)$ do que para homens $(26 \%)$, embora sem significância estatística para a diferença entre essas proporções, conforme se observa na Tabela 3. Para os grupos etário, por sua vez, há uma maior concentração dos indivíduos que já pensaram em se mudar por causa da seca nos grupos de 20-24 anos $(36,5 \%)$ e de $25-34$ anos (32\%). O teste Qui-quadrado mostrou que a diferença entre as proporções para os grupos etários selecionados apresentou significância estatística de 5\%. Nesse sentido, os mais jovens tendem a considerar a mudança de residência habitual com maior frequência que os mais velhos, de acordo com os resultados encontrados em pesquisas anteriores (Koubi, Stoll, Spilker, 2016; Koubi et al., 2016). 
Tabela 3 - Seridó Potiguar: indivíduos entrevistados na área urbana segundo a percepção sobre a decisão de migração induzida pela seca, o grupo etário e sexo, 2017

\begin{tabular}{|c|c|c|c|}
\hline \multirow{2}{*}{ Gr. Etário } & \multicolumn{3}{|c|}{ Já pensou em se mudar por causa da seca? } \\
\hline & $\operatorname{Sim}(\%)$ & Não (\%) & \\
\hline $15-19$ & 25,8 & 74,2 & \multirow{8}{*}{$\begin{array}{c}\text { Qui- } \\
\text { quadrado } \\
14,38^{* *}\end{array}$} \\
\hline $20-24$ & 36,5 & 63,5 & \\
\hline $25-34$ & 32,0 & 68,0 & \\
\hline $35-44$ & 23,9 & 76,1 & \\
\hline $45-54$ & 30,3 & 69,7 & \\
\hline $55-64$ & 23,7 & 76,3 & \\
\hline $65 e+$ & 18,9 & 81,1 & \\
\hline Missing & 20,0 & 80,0 & \\
\hline Sexo & $\operatorname{Sim}(\%)$ & Não (\%) & \\
\hline Masculino & 26,0 & 74,0 & \multirow{3}{*}{$\begin{array}{c}\text { Qui- } \\
\text { quadrado } \\
0.067\end{array}$} \\
\hline Feminino & 27,0 & 73,0 & \\
\hline Missing & 0 & 100,0 & \\
\hline Total & 26,7 & 73,3 & \\
\hline
\end{tabular}

Fonte: Survey Seridó Potiguar (2017), N=1.064 (Gr. Etário, missing =

5; Sexo, missing $=3$ ). ${ }^{* * *} \mathrm{p}<0.01,{ }^{* *} \mathrm{p}<0.05,{ }^{*} \mathrm{p}<0.1$.

\section{Considerações finais}

Como observada por meio da análise conduzida nesse trabalho, a intenção de se mudar por causa da seca não foi uma alternativa vislumbrada pela maior parte da população urbana do Seridó Potiguar. Isso não torna a mobilidade uma estratégia de adaptação menos importante em áreas urbanas no contexto das secas, mas só delineia a necessidade de considerar outros mecanismos de adaptação além da migração, inclusive outras formas de mobilidade sem desvinculação com o lugar de residência habitual. Cabe destacar, ademais, pelo menos três contribuições desse artigo: 1) definição de formas de mobilidade em função do tempo e do espaço como estratégias de adaptação às secas; 2) subsídios de um modelo conceitual onde as diferentes formas de mobilidade interagem com a percepção da mobilidade como estratégia de adaptação e 3) fornecimento de um mecanismo para entender como a intenção de mobilidade como estratégia de adaptação pode estar relacionada com a forma de mobilidade praticada pelo indivíduo.

Em síntese, os resultados mostraram que dentre a população urbana residente no Seridó Potiguar os não naturais do município de residência, os que efetuam formas de mobilidade de distâncias mais longas, os que praticam mais de uma forma de mobilidade, os que retornam diariamente para casa, os que passam 
menos tempo em dias fora do domicílio de residência habitual e os mais jovens tendem a considerar mais a mudança de residência habitual por causa da seca em comparação aos demais grupos. As mulheres também têm mais intenção de se mudar por causa da seca do que os homens, embora sem significância estatística para a diferença entre essas proporções. De certo modo, como a literatura já havia apontado, já esperava-se que os deslocamentos da seca fossem mais de caráter temporário e de curta distância em detrimento de outras formas de mobilidade como as que envolvem o cruzamento de fronteiras internacionais e de caráter permanente como a migração internacional ou inter-regional, embora pouco possa-se afirmar até que ponto essas estratégias de "andar pelos sertões" refletem escolhas ou oportunidades. Por outro lado, a experiência com a mobilidade poderá influenciar a intenção de mobilidade em áreas urbanas da região do Seridó em contextos de secas no futuro.

É necessária, portanto, uma maior sensibilidade crítica ao categorizar e estudar esses fluxos populacionais enquanto migrantes ambientais, ou melhor dizendo, os deslocamentos da seca. Em alguns casos a mobilidade não é uma alternativa viável porque ela exige recursos e pode ser onerosa em termos psicológicos e econômicos no caso da mobilidade de longa distância, ao romper laços com o domicílio de origem por exemplo. A seca, por sua vez, pode intensificar processos de exclusão ao exaurir recursos necessários a mobilidade. Portanto, é importante que os formuladores de políticas públicas tenham um olhar específico sobre as populações afetadas pelas secas para criar condições por meio de políticas específicas para que os indivíduos tenham a oportunidade de escolha por estratégias de adaptação, dentre elas a mobilidade.

Por fim, uma agenda de pesquisa que leve em conta essas singularidades para estudar as condições de vida da população nordestina no contexto das estiagens precisa ser construída. Os avanços desse trabalho, embora que ainda sejam de caráter preeliminar, contribuem para a consolidação e ampliação de uma agenda de pesquisa, a "demografia da seca", que foi sugerida pelo demógrafo e sociólogo Ricardo Ojima (2012, p. 261) o que corresponderia a uma modalidade sertaneja do que o demógrafo Daniel Hogan chamou de Demografia Ambiental (Freire de Mello, Sathler, 2015). Desse modo, o semiárido nordestino possui um campo fértil para o debate sobre as sinergias entre população, ambiente e desenvolvimento que precisa ser melhor explorado pelos estudiosos de população.

Agradecimentos: $\mathrm{O}$ autor agradece o suporte financeiro à pesquisa que originou esse artigo, através da Rede Brasileira de Pesquisas em Mudanças Climáticas (Rede Clima, FINEP/MCTI), do Inter-American Institute for Global Change Research (IAI) e do Conselho Nacional de Desenvolvimento Científico e Tecnológico (CNPq, 447688/2014-6 e 306567/2016-4); e a agência de fomento Coordenação de Aperfeiçoamento de Pessoal de Nível Superior (Capes/PROEX) pela bolsa de doutorado. 


\section{Referências bibliográficas}

AB'SÁBER, Aziz N. Sertões e sertanejos: uma geografia humana sofrida. Estudos Avançados, v. 13, n. 36, p. 7-59, 1999.

BARBIERI, Alisson F. Mudanças climáticas, mobilidade populacional e cenários de vulnerabilidade para o Brasil. REMHU, Revista Interdisciplinar da Mobilidade Humana, v. 36, p. 95-112, 2011.

BARBIERI, Alisson F.; GUEDES, Gilvan; CORREIA, Isac; OJIMA, Ricardo. Population mobility and adaptation to droughts in the Brazilian semi-arid. Proceedings of Meeting of Population Association of American - PAA. Austin, Texas: PAA, v. 1, p. 1-15, 2019.

BERKES, Fikret; JOLLY, Dyanna. Adapting to climate change: social-ecological resilience in a Canadian western arctic community. Conserv. Ecol., v. 5, n. 2, p. 1-15, 2002.

BLACK, Richard; ADGER, W. Neil; ARNELL, Nigel W.; DERCON, Stefan; GEDDES, Andrew; THOMAS, David. The effect of environmental change on human migration. Glob. Environ. Change, v. 21, p. S3-S11, 2011.

CALL, Maia; GRAY, Clark; YUNUS, Mohammad; EMCH, Michael. Disruption, not displacement: environmental variability and temporary migration in Bangladesh. Glob. Environ. Change, v. 46, n. 3, p. 157-165, 2017.

CAMPOS, Ricardo S. Living with drought: a study of spatial mobility in semi-arid Northeast Brazil. PhD Thesis (degree of Doctor of Philosophy). School of Geography, Planning and Environmental Management, University of Queensland - UQ, 2015.

CORREIA, Isac A. Vulnerabilidade e adaptação no Seridó Potiguar: a (i)mobilidade e estratégias domiciliares. Dissertação (Mestrado em Demografia). Departamento de Demografia e Ciências Atuariais - DDCA/UFRN. Natal/RN: UFRN, 2018.

CORREIA, Isac A.; Ojima, Ricardo. Migração e (i)mobilidade no Nordeste brasileiro: adaptação para quem? Revista Brasileira de Gestão e Desenvolvimento Regional, v. 15, n. 5, p. 138-151, 2019.

CORREIA, Isac A.; OJIMA, Ricardo; BARBIERI, Alisson Flávio. Emigração e transferências monetárias como estratégias de adaptação às secas no Seridó Potiguar. REMHU, Revista Interdisciplinar da Mobilidade Humana, v. 28, n. 59, p. 187-207, 2020.

FARIA, Oswaldo L. Os açudes dos sertões do Seridó. Natal/RN: Sebo Vermelho, 2012.

FINDLEY, Sally E. Does drought increase migration? A study of migration from rural Mali during the 1983-1985 drought. Int. Migr. Rev., v. 28, n. 3, p. 539-553, 1994.

FRANKENBERG, Elizabeth; LAURITO, Maria; THOMAS, Duncan. The demography of disasters. In: WRIGHT, James D. (ed.). International Encyclopedia of the Social and Behavioral Sciences. Oxford, UK: Elsevier, 2015, p. 559-565.

FREIRE DE MELLO, Leonardo; SATHLER, Douglas. A demografia ambiental e a emergência dos estudos sobre população e consumo. R. bras. Est. Pop., v. 32, n. 2, p. 357-380, 2015.

GONÇALVES, Alfredo José. Migrações internas: evoluções e desafios. Estudos Avançados, v. 15, n. 43, p. 173-184, 2001.

IBGE - Instituto Brasileiro de Geografia e Estatística. Censo Demográfico do Brasil. Rio de Janeiro: IBGE, 2010. 
GRAY, Clark L.; BILSBORROW, Richard. Environmental influences on human migration in rural Ecuador. Demography, v. 50, p. 1217-1241, 2013.

GRAY, Clark L.; MUELLER, Valerie. Drought and population mobility in rural Ethiopia. World Development, v. 40, n. 1, p. 134-145, 2012.

HENRY, Sabine; SCHOUMAKER, Bruno; BEAUCHEMIN, Cris. The impact of rainfall on the first out-migration: A multi-level event-history analysis in Burkina Faso. Popul. Environ., v. 25, n. 5, p. 423-460, 2004.

HIRSCHMAN, Albert O. Journeys toward progress: studies of economic policy-making in Latin America. New York: The Twentieth Century Fund, 1963.

HUGO, Graeme. Environmental concerns and international migration. Int. Migr. Rev., v. 30, n. 1, p. 105-131, 1996.

HUNTER, Lori M. et al. Rural outmigration, natural capital, and livelihoods in South Africa. Popul. Space Place, v. 20, n. 5, p. 402-420, 2014.

HUNTER, Lori M.; LUNA, Jessie K.; NORTON, Rachel M. Environmental dimensions of migration. Annu Rev Sociol, v. 41, n. 6, p. 1-21, 2015.

KNIVETON, Dominic et al. Climate change and migration: improving methodologies to estimate flows. Brighton, UK: International Organization for Migration, 2008.

KOUBI, Vally; STOLL, Sebastian; SPILKER, Gabriele. Perceptions of environmental change and migration decisions. Climatic Change, v. 138, p. 439-451, 2016.

$\mathrm{KOUBI}$, Vally et al. The role of environmental perceptions in migration decision-making: evidence from both migrants and non-migrants in five developing countries. Popul. Environ., v. 38, n. 2, p. 134-163, 2016.

MACÊDO, Muirakytan K. A penúltima versão do Seridó: uma história do regionalismo seridoense. Natal/RN; Campina Grande/PB: EDUEPB/EDUFRN, 2012.

MORAIS, lone R Diniz. Seridó norte-rio-grandense: reestruturação e planejamento regional. In: Encontro da Associação Nacional de Pós-Graduação e Pesquisa em Planejamento Urbano e Regional, 11. Anais... Salvador/BA: ANPUR, 2005.

MUELLER, Valerie; GRAY, Clark L.; KOSEC, Katrina. Heat stress increases long-term human migration in rural Pakistan. Nat. Climate Change, v. 4, n. 3, p. 182-185, 2014.

NAWROTZKI, Raphael J.; DeWAARD, Jack. Climate shocks and the timing of migration from Mexico. Popul. Environ., v. 38, n. 1, p. 72-100, 2016.

OJIMA, Ricardo. Mobilidade populacional, condições de vida e desenvolvimento no semiárido brasileiro: por uma demografia da seca. In: GUEDES, Gilvan Ramalho; OJIMA, Ricardo (orgs.). Território, Mobilidade Populacional e Ambiente. Univale/ CBH-Doce: Governador Valadares, MG, 2012.

OJIMA, Ricardo. Urbanização, dinâmica migratória e sustentabilidade no semiárido nordestino: o papel das cidades no processo de adaptação ambiental. Cad. Metrop., v. 15, n. 29, p. 35-54, 2013.

OJIMA, Ricardo; COSTA, José Vilton; CALIXTA, Renata Kissya. "Minha vida é andar por esse país...": a emigração recente no semiárido setentrional, políticas sociais e meio ambiente. REMHU, Revista Interdisciplinar da Mobilidade Humana, v. 43, p. 149-167, 2014. 
OJIMA, Ricardo; FUSCO, Wilson. Migração no semiárido setentrional: dinâmica recente, retorno e políticas sociais. MEDIAÇÕES - Revista de Ciências Sociais, v. 22, p. 325-349, 2017.

McCULLAGH, Peter; NELDER, John. Generalized Linear Models. New York: Chapman and Hall/CRC, 1989.

RIOS, Kênia S. Isolamento e poder: Fortaleza e os campos de concentração na seca de 1932. Fortaleza: Imprensa Universitária, 2014.

SCHADE, Janette; FAIST, Thomas; MCLEMAN, Robert. Conclusion: inequality and migration as adaptation: where do we go from here? In: MCLEMAN, Robert; SCHADE, Janette; FAIST, Thomas (eds.). Environmental migration and social inequality. Dordrecht: Springer, 2015, p. 203-228.

SELLERS, Samuel; GRAY, Clark. Climate shocks constrain human fertility in Indonesia. World Development, v. 117, p. 357-369, 2019.

SHERBININ, Alex et al. Rural household demographics, livelihoods and the environment. Glob. Environ. Change, v. 18, n. 1, p. 38-53, 2008.

ZANDER, Kerstin K.; RICHERZHAGEN, Carmen; GARNETT, Stephen T. Human mobility intentions in response to heat in urban South East Asia. Glob. Environ. Change, v. 56, p. 18-28, 2019. 